\title{
Propagation of light through ship exhaust plumes
}

\author{
M. van Iersel ${ }^{* a}$, A. Mack ${ }^{b}$, A.M.J. van Eijk ${ }^{\text {a }}$, and H.M.A. Schleijpen ${ }^{\text {a }}$ \\ ${ }^{a}$ TNO, Oude Waalsdorperweg 63, 2597 AK The Hague, The Netherlands \\ ${ }^{\mathrm{b}}$ TNO, Stieltjesweg 1, $2628 \mathrm{CK}$ Delft, The Netherlands
}

\begin{abstract}
Looking through the atmosphere, it is sometimes difficult to see the details of an object. Effects like scintillation and blur are the cause of these difficulties. Exhaust plumes of e.g. a ship can cause extreme scintillation and blur, making it even harder to see the details of what lies behind the plume.

Exhaust plumes come in different shapes, sizes, and opaqueness and depending on atmospheric parameters like wind speed and direction, as well as engine settings (power, gas or diesel, etc.). A CFD model is used to determine the plume's flow field outside the stack on the basis of exhaust flow properties, the interaction with the superstructure of the ship, the meteorological conditions and the interaction of ship's motion and atmospheric wind fields. A modified version of the NIRATAM code performs the gas radiation calculations and provides the radiant intensity of the (hot) exhaust gases and the transmission of the atmosphere around the plume is modeled with MODTRAN. This allows assessing the irradiance of a sensor positioned at some distance from the ship and its plume, as function of the conditions that influence the spatial distribution and thermal properties of the plume. Furthermore, an assessment can be made of the probability of detecting objects behind the plume.

This plume module will be incorporated in the TNO EOSTAR-model, which provides estimates of detection range and image quality of EO-sensors under varying meteorological conditions.
\end{abstract}

Keywords: Exhaust plumes, atmospheric propagation, transmission, radiance, IR signatures, modelling

\section{INTRODUCTION}

Nowadays the limiting factor of imaging systems deployed in the field is often not of a technological nature. Usually it is the atmosphere between an object and the imaging sensor that degrades the performance of the imaging system. Molecules and aerosols in the atmosphere cause scattering and absorption of radiation, resulting in transmission losses. Spatial and temporal fluctuations in the temperature and humidity of the atmosphere induce inhomogeneities in the refractive index, which in turn are responsible for the blurring and scintillation in an image. The presence of non-natural objects in the atmosphere may lead to (a) further degradation of image quality. Exhaust plumes of aircraft or ships alter the natural composition of the atmosphere by introducing a different mixture of gases at higher temperature and density. Mixing of these hot exhaust gases with the surrounding air are the cause of turbulence effects in the so-called plume region, which extends to some distance from the exhaust. Typical values of the refractive index structure parameter, $\mathrm{C}_{\mathrm{n}}{ }^{2}$, for an exhaust plume (over a relatively short path) are of the order of $10^{-10} \mathrm{~m}^{-2 / 3}$. The presence of an exhaust plume can thus cause severe blurring and scintillation in optical paths that intersect with the plume region, in addition to enhanced transmission losses due to the hot and concentrated gases in the plume. This is an important effect for e.g. directed infrared countermeasure (DIRCM) systems, which use a directed laser to counter a threat from e.g. a missile. A good overview of the study into laser beam propagation through a turbulent region of the atmosphere, like a jet engine plume, is given in [1] [2] [3].

This paper focuses on the propagation of light through ship exhaust plumes. While the temperatures in these ship exhaust plumes are not as high as the plumes of jet engines and the effects in the image are thus less severe, the plume temperature is still a few hundred degrees higher than the surrounding atmosphere resulting in a reasonable loss of transmission. Engine settings and type of engine (diesel or gas turbine), speed and engine power have an influence on the size of the exhaust plume and on the concentration of species (predominantly $\mathrm{CO}_{2}, \mathrm{H}_{2} \mathrm{O}$, and $\mathrm{CO}$ ) present in it. Since the plume exit velocity is lower than that of a jet plume, the interaction with external parameters such as the wind field becomes more prominent. This does not only apply to the larger-scale plume dispersion in the atmosphere, but also to the initial dynamics that are influenced by the interaction of the wind field with the ship's superstructure. An analysis of the influence of the

*miranda.vaniersel@tno.nl

Laser Communication and Propagation through the Atmosphere and Oceans III, edited by Alexander M. J. van Eijk, Christopher C. Davis, Stephen M. Hammel, Proc. of SPIE Vol. 9224, 92240T ( 2014 SPIE CCC code: $0277-786 X / 14 / \$ 18 \cdot$ doi: $10.1117 / 12.2063372$ 
aforementioned parameters on the characteristics of the plume, such as spatial coverage and infrared (IR) signature of the exhaust plume will be presented. To calculate the IR signature of the exhaust plume one needs to calculate the radiation by the gas in the exhaust plume as well as the transmission through the plume. The gas radiation calculations are performed by a modified version of NIRATAM [4]. A computational fluid dynamics (CFD) model is used to provide information on the 3D flow field of the exhaust plume (spatial coverage, gas concentrations, densities, and temperatures), which then can be used as input to the gas radiation calculations. The exhaust plume model will ultimately become part of the EOSTAR Pro model suite developed by TNO The Hague in cooperation with the Royal Netherlands Navy and SPAWAR Systems Center Pacific San Diego [5][6][7]. The EOSTAR model aims at providing an end-to-end solution for the assessment of sensor performance against a specific threat in a specific (maritime) environment. Thus, the radiant intensities of the target [8] and its background [9] are evaluated as function of the environmental conditions [10][11]. In our methodology we do not intend to couple the CFD calculations directly to EOSTAR, like is done in other ship signature prediction models ShipIR [12].

This paper discusses the different steps in the methodology to include the radiation of and transmission through the exhaust plume into EOSTAR in section 2. An analysis of the influence of several parameters on the IR signature of the exhaust plume will be presented in section 3. Some discussion and conclusions will be given in section 4 .

\section{METHODOLOGY}

The methodology to include radiation of an exhaust plume and transmission through this plume into EOSTAR consists of different steps, which are explained in more detail in the next subsections. The first step is to use CFD calculations to determine the 3D flow field of the plume. This flow field serves as input to the gas radiation calculations performed by the modified version of NIRATAM. The output of these calculations will be the IR signature of the exhaust plume. This signature will be coupled to EOSTAR. At the moment there is no direct coupling between the different models.

\subsection{CFD calculations}

Computational Fluid Dynamics (CFD) is used to model the 3D flow field around a ship including its exhaust plume. Included in these calculations are the turbulent mixing effects of the hot exhaust gases with the ambient air containing mainly $\mathrm{CO}_{2}, \mathrm{H}_{2} \mathrm{O}$, and $\mathrm{CO}$ and the interaction with the ship geometry itself. The CFD model consists of the Reynolds Averaged Navier-Stokes (RANS) equations with a standard k- $\varepsilon$ closure for turbulence, which are solved using Ansys Fluent [13]. In order to minimize the number of transport equations that need to be solved, the exhaust gas was taken to consist of air, having a temperature of $633 \mathrm{~K}$. The temperature of the surrounding environment was set to $300 \mathrm{~K}$. Since we are only interested in the exhaust plume, the local part of the numerical CFD domain containing the exhaust plume is interpolated on a mesh of $100 \times 100 \times 50$ points after conclusion of the run for the radiation analysis. For the studies presented in this paper, a simplified geometry of the Royal Netherlands Navy M-frigate was used (see Figure 1). This simplified geometry was used since the focus of the present investigation is the main interaction of the flowfield surrounding the ship with the exhaust plume neglecting local flow features.

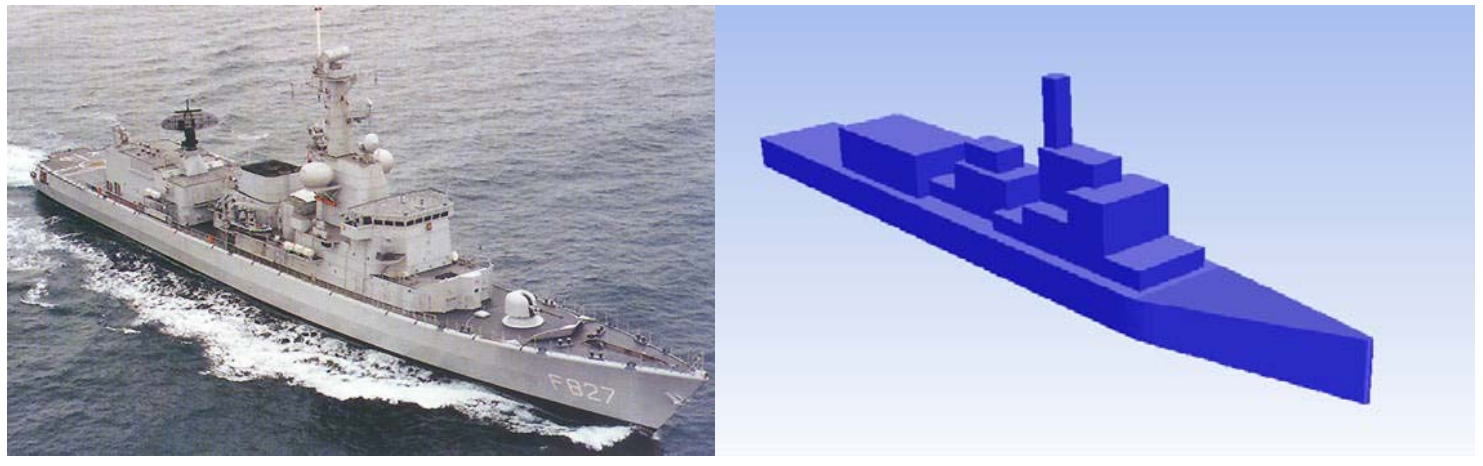

Figure 1 Dutch M-frigate and the simplified geometry used in the CFD calculations.

Input parameters to the CFD calculations are the wind speed and direction, ship speed and heading, engine power, the temperature of the exhaust gas, the speed of the exhaust gas at the exit plane and a geometrical representation of the ship's superstructure. The speed (vector) is a combination of the ship's speed (vector) and the wind speed (vector). From 
experimental data, the exhaust gas composition at the exhaust exit plane is known and since no further chemical reactions take place the mixing of the exhaust gas species can be considered as passive. By this, the species concentration can be derived from the temperature distribution of the plume which serves as an indicator for the mixing process. The exhaust gas is modelled as one component (air) and the concentrations of the relevant gasses $\left(\mathrm{CO}_{2}, \mathrm{H}_{2} \mathrm{O}\right.$, and $\mathrm{CO}$ ) are obtained from the temperature distribution, which is done assuming identical Schmidt and Prandtl numbers. By this, the thermal and mass diffusion processes are correlated similarly with respect to the momentum diffusion. The results have been validated with previous CFD simulations, where all mass and thermal diffusion processes were included. From these investigations, the correction factor $F$ in Eqn. (1) was derived.

Only three gasses $\left(\mathrm{CO}_{2}, \mathrm{H}_{2} \mathrm{O}\right.$, and $\left.\mathrm{CO}\right)$ are considered as constituents of the plume. The output parameters of the calculations are the position coordinates $(\mathrm{x}, \mathrm{y}, \mathrm{z})$, and for each coordinate, the pressure, density and temperature of the flowfield. The molar fractions of $\mathrm{H}_{2} \mathrm{O}, \mathrm{CO}_{2}$, and $\mathrm{CO}$, denoted by $\mathrm{C}$, are derived from the temperature field $\mathrm{T}$ using

$$
C=C_{\max }\left(\frac{T-T_{r e f}}{T_{\max }-T}\right)^{F},
$$

with the reference temperature $\mathrm{T}_{\text {ref }}=300 \mathrm{~K}$ being the background temperature of the atmosphere, the release temperature of the exhaust is $\mathrm{T}_{\max }=633 \mathrm{~K}$ at $100 \%$ power setting. The correction factor is $\mathrm{F}=1.005$ and the maximum molar fractions, $\mathrm{C}_{\max }$, for the different species are given by $\mathrm{C}_{\max }\left(\mathrm{CO}_{2}\right)=0.037, \mathrm{C}_{\max }(\mathrm{CO})=0.00015$, and $\mathrm{C}_{\max }\left(\mathrm{H}_{2} \mathrm{O}\right)=0.037$. The maximum exhaust temperature and molar fractions are measured values from the exhaust gas of the ship at the top of the stack. Here, the maximum molar fractions are taken as constant since no load dependent values were available. The maximum exhaust temperatures are taken according to measured data.

\subsection{Plume radiation calculations}

The radiant intensity of the exhaust plume is calculated using a modified version of the NATO Infrared Air Target Model (NIRATAM) [4]. The major modification consists of replacing the (2D) rotation-symmetric exhaust plume provided by NIRATAM's sub-model N-plume by the (asymmetric (3D)) ship exhaust plume provided by the CFD code discussed in the previous section. Furthermore, the aircraft model in NIRATAM was replaced by a rudimentary ship model. The gas radiation calculations form, also in this modified version, the core of NIRATAM.

The radiant intensity of the exhaust plume results from three effects: (1) spectral emission lines determined by the composition and temperature of the gases; (2) broadband (Planck) radiation determined by the temperature of the gases; (3) (spectral) absorption of the radiance by the plume itself. The spectral lines denote transitions between the vibrationalrotational energy levels of a molecule. Each vibration in the molecule gives rise to a band of spectral lines with energies corresponding to the common vibrational transition energy offset by the various rotational sub-transitions. The fundamental lines are broadened by the Doppler effect and by collisions between the molecules. Ideally, the radiant intensity is calculated by a summation of all individual lines, but this process is time-consuming and often the transition cross-sections are not precisely known. Therefore, NIRATAM uses band-line models in which the spectrum is divided in several intervals / bands. A single-line group (SLG) model or a multi-line group (MLG) model can be used to calculate the spectrum. In a SLG model all lines within an interval are treated as if they represent a single line. In a MLG model the lines in an interval are divided into groups such that all lines in a group have similar strengths. In both models the Curtis-Godson approximation is used to replace the inhomogeneous concentration of the species in an interval with a homogeneous concentration.

For a specified sensor-plume viewing geometry, NIRATAM divides the exhaust plume in segments and passes a number of rays through the plume along the line of sight of the sensor. For each segment the contribution to the radiation in the wavelength band of the sensor is added to a running sum of the total radiation along the line of sight. The radiation transfer equations and spectral information are taken from [14]. The Planck radiation and the spectral line contributions of the molecules $\mathrm{H}_{2} \mathrm{O}, \mathrm{CO}_{2}$, and $\mathrm{CO}$ in the exhaust plume are evaluated. Also included in the calculations are the molecules $\mathrm{H}_{2}$, $\mathrm{N}_{2}$, and $\mathrm{O}_{2}$. While these do not yield vibrational-rotational spectral emission line because of the absence of an electric dipole moment, they contribute to the line broadening of the spectral lines of the other molecules. Furthermore, the transmission through the exhaust plume is calculated as well as the transmission of the radiation of the exhaust plume through the atmosphere. The total signal received at the sensor consists of two parts; the radiation of the plume transmitted through the atmosphere over a path between plume and sensor and the transmission of the background radiation through the atmosphere over a path between background and plume, then through the exhaust plume, and finally the transmission through the atmosphere over a path between the plume and the sensor. 
The 3D flow fields calculated with the CFD code are input for the calculations with the modified version of NIRATAM. Among the other input parameters are the positions of the exhaust plume and sensor, the background temperature and emissivity, wavelength or wavenumber interval, and a characterization of the atmosphere. The atmospheric transmission can be calculated using an internal LOWTRAN module, or by an external coupling to MODTRAN. One can use the five standard pre-defined atmospheric models, or use a file to specify the atmosphere. It is also possible to calculate the radiation of the plume, without any atmosphere present. A complete overview of the input parameters is given in [15]. As an output NIRATAM can produce image files containing the radiant intensity, radiance, or apparent temperature as well as a spectral radiance file containing the intrinsic or apparent radiance for the wavelength or wavenumber interval. The image file is only spatially resolved and provides a $2 \mathrm{D}$ radiance image of the plume viewed by the sensor. The spectral radiance file contains a spectral table of plume radiance and plume transmission. This table is spatially not resolved and thus represents the "overall" radiance and transmission as viewed by the sensor.

\subsection{EOSTAR}

As already mentioned, the plume module will be coupled to the Electro-Optical Signal Transmission And Ranging (EOSTAR) model. EOSTAR evaluates the performance of an electro-optical sensor against targets in a scene under varying meteorological conditions. One of the central elements in EOSTAR is the Snell's Law Ray Tracing Scheme (SLARTS) [16], which provides the 'sensor ray trajectories' that are the basis for evaluating the effects of atmospheric refraction and path-integrated effects (e.g., transmission, radiance, blur, and scintillation) on the image. The ray trajectories represent the observation paths of the individual sensor pixels and the trajectories can thus be considered as the axes of the instantaneous field of view (IFOV) of the single detector elements of the sensor. Each trajectory is launched from the center of this pixel, crosses the middle of the optics and starts its propagation path in the atmosphere at the position and at the azimuth and elevation angles corresponding to the particular detector element. The fan of ray trajectories covers the field-of-view of the sensor.

In order to calculate the ray trajectories through the atmosphere, EOSTAR first evaluates the fields of pressure, temperature and humidity, using (a combination of) Monin-Obukhov similarity theory, radiosonde observations and numerical weather prediction data [10][11], and from these, the field of refractive index. The fields of pressure, temperature and humidity, as well as various other parameters that characterize the atmosphere, are also used to drive MODTRAN for the evaluation of molecular transmission, and ANAM for the evaluation of aerosol extinction in a maritime environment. Finally, the field of the refractive index structure-function parameter $C_{n}^{2}$ can be evaluated, which quantifies turbulence effects (scintillation, blur) on the image.

The trajectories are constructed as a series of small steps (typically $50 \mathrm{~m}$ ). At each step, the local ray direction and local refractive index gradient are used to evaluate the change in ray direction due to refraction in a spherically stratified atmosphere. At the same time, the transmission losses due to molecular and aerosol extinction are evaluated over the step, and the overall transmission from the sensor to the current ray position. Neighboring rays are used to evaluate the local geometric distortion, i.e., the phenomenon that refractive effects cause the pixel IFOV to deviate from $R^{2}$. The geometric gain factor and the transmission losses together specify the propagation factor, i.e., the transfer function for radiant intensity between the sensor and the ray position. When an object (e.g., a ship) with a specific radiant intensity is positioned in the scene, the ray tracer thus provides the distribution of detector IFOVs over the target and all the information required to evaluate the radiant intensity of the target on the sensor.

The coupling of the exhaust plume to EOSTAR will be, in first instance, done by file transfer. Intermediate results of the modified version of NIRATAM are written to file. These results contain the spatially and spectrally resolved radiation of the exhaust plume. These results are calculated without any atmosphere present in NIRATAM and with the same sensor characteristics and sensor - target geometry as EOSTAR will use. EOSTAR reads in the file and places the exhaust plume on top of the exhaust of the ship. The atmosphere, sensor characteristics, and sensor - target geometry are defined within EOSTAR, and the latter two should be equal to the settings used in NIRATAM. EOSTAR then calculates the radiant intensity of the ship including the exhaust plume on the sensor.

EOSTAR provides a synthetic image of the scene as perceived by the sensor as output. Another output of EOSTAR consists of range-height and range-azimuth coverage diagrams of quantities of interest (e.g., detection range). When building coverage diagrams, EOSTAR takes target constraints into account, e.g., the constraint that a ship must be at the surface, whereas an aircraft can be at any altitude. 


\section{RESULTS}

The size, temperature distribution and shape of an exhaust plume are influenced by atmospheric parameters like the (air) temperature, wind speed and direction, as well as by engine settings like engine power, speed, and type of engine. These parameters also have an influence on the radiant intensity of the exhaust plume and the transmission through the plume. The sections below addresses the influence of several of these parameters on the radiant intensity and transmission through the exhaust plume.

In all cases a model of the Royal Netherlands Navy M-frigate is used to calculate the exhaust plumes of the ship (see Figure 1). The speed vector, $\mathbf{v}$, is a combination of the ship's speed vector and the wind speed vector. The combined speed vector is denoted by a length $\mathrm{v}$ and a direction $\theta$. The ship is assumed to sail in a heading of $0^{\circ}$. A wind direction of $0^{\circ}$ is defined as head on to the bow of the ship. A homogeneous background with a temperature of $300 \mathrm{~K}$ is used in the simulations. The sensor is positioned behind and to the left of the ship, with a range $\mathrm{R}=2 \mathrm{~km}$.

\subsection{Output of modified version of NIRATAM for the case $v=15 \mathrm{~m} / \mathrm{s}, \theta=0^{\circ}$, engine power $=100 \%$}

In this case, which will serve as a reference later on, the combined speed is $\mathrm{v}=15 \mathrm{~m} / \mathrm{s}$ and the direction $\theta=0^{\circ}$ (head on). The engine power is set to $100 \%$ and the exhaust gas temperature at the exit of the stack is $633 \mathrm{~K}$. The calculations are performed without any atmosphere present. The modified version of NIRATAM can give as output a radiant intensity image, as well as the apparent or intrinsic radiance, and the contrast intensity of the object (plume) against the background. An example of a radiance map is shown in Figure 2 (a). Note that these images are spatially resolved, but not spectrally. The example shows the radiance integrated over the band of $3.0-5.0 \mu \mathrm{m}(3333-2000 \mathrm{~cm}-1)$. Figure 2 (b) the contrast irradiance spectrum is shown as calculated for the wavelength band of $2.0-14.0 \mu \mathrm{m}(5000-715 \mathrm{~cm}-1)$. Note that this image is not spatially resolved, meaning that an overall plume radiance and transmission is calculated. The spectrum Figure 2 shows the peaks that correspond to the gases $\mathrm{CO} 2, \mathrm{CO}$, and $\mathrm{H} 2 \mathrm{O}$. Also visible are several wavelength bands where the contrast intensity is zero. Since no atmosphere was taken into account, the bands with zero contrast intensity correspond to wavelength bands were no radiation is produced by the plume.

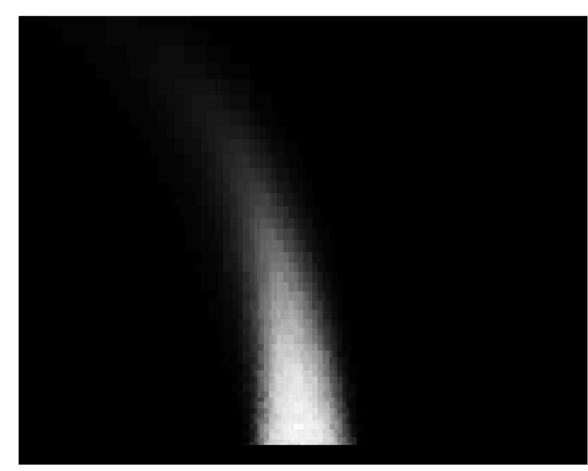

(a)

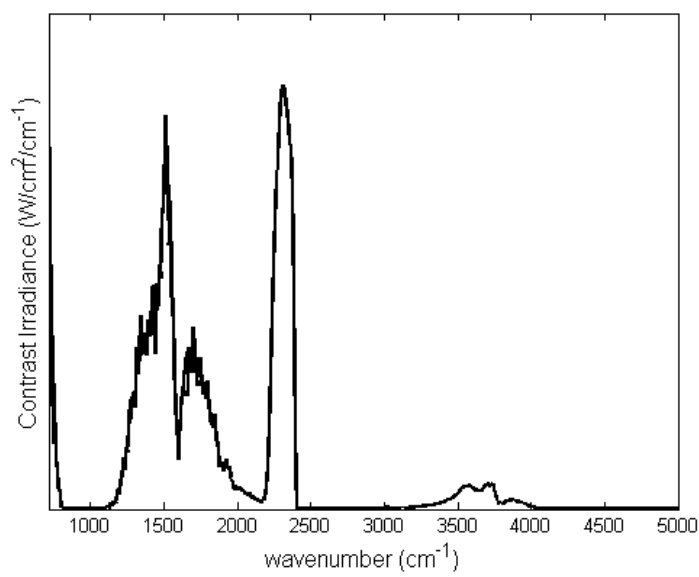

(b)

Figure 2 (a) Radiance map of the exhaust plume for 3.0 - $5.0 \mu \mathrm{m}$ and (b) the contrast irradiance spectrum of the plume for $2.0-14.0 \mu \mathrm{m}$. (ignoring atmospheric effects)

As mentioned above, Figure 2 was generated in the absence of an atmosphere. The modified version of NIRATAM also has the option to calculate the radiance of the plume with an atmosphere present. There are two options to model the atmosphere; the first one uses an internal routine based on LOWTRAN, in the second option MODTRAN is coupled to the modified version of NIRATAM. In our case we have used version 4.3.1 of MODTRAN. In both options several predefined models can be used. For the cases presented here a mid-latitude summer model was used with a marine aerosol model and $23 \mathrm{~km}$ of visibility.

Figure 3 (a) shows the contrast irradiance spectrum for $2.0-14.0 \mu \mathrm{m}\left(5000-715 \mathrm{~cm}^{-1}\right)$ of the exhaust plume calculated with no atmosphere (black line), the atmosphere calculated with LOWTRAN (red line) and with MODTRAN (blue line). In Figure 3 (b) we have zoomed in on the contrast irradiance spectra calculated with an atmosphere present. It 
demonstrates the superior resolution of the MODTRAN calculation, and suggests that the external coupling with MODTRAN should be preferred for detailed calculations. One should realize that, for a thorough calculation, the spectral resolution of the atmospheric transmission calculations should match the spectral resolution of the radiance calculations.

Figure 3 shows that the plume generates a lot of radiation in the wavelength band of $5.5-7.5 \mu \mathrm{m}\left(1333-1818 \mathrm{~cm}^{-1}\right)$ and that the atmosphere absorbs almost all the radiation in this wavelength band. Only around the edges of the plume emission band the radiation partially traverses the atmosphere. A similar effect occurs around $4.1-4.5 \mu \mathrm{m}\left(2222-2439 \mathrm{~cm}^{-1}\right)$. Around these wavelengths the well-known blue and red spike caused by $\mathrm{CO}_{2}$ produce a lot of radiation, which is for a large part absorbed by $\mathrm{CO}_{2}$ in the atmosphere. Also here the radiation around the edges of this domain comes partially through the atmosphere.

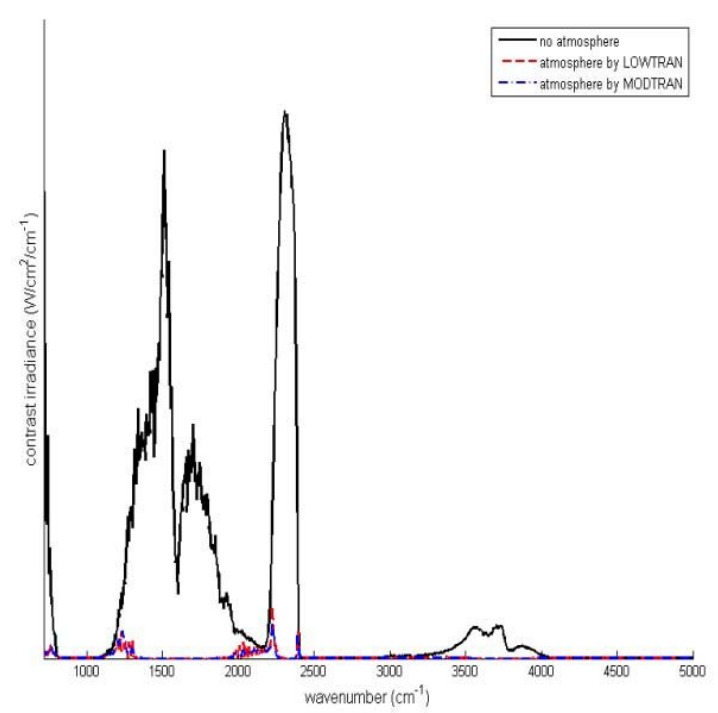

(a)

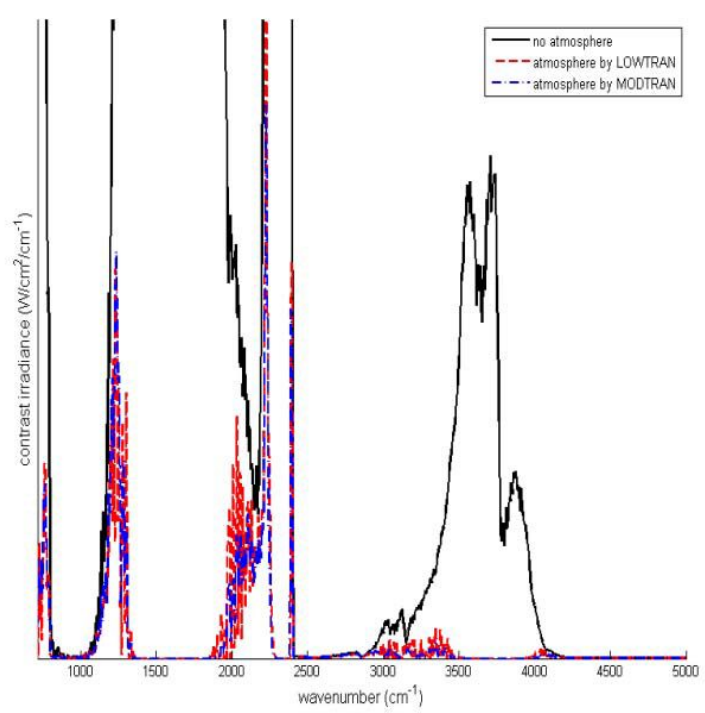

(b)

Figure 3 (a) Contrast irradiance spectrum of an exhaust plume without atmosphere (black line) and with the atmosphere calculated with LOWTRAN (red line) or MODTRAN (blue line) for $2.0-14.0 \mu \mathrm{m}$. (b) Zoomed in on the contrast irradiance spectra calculated with an atmosphere.

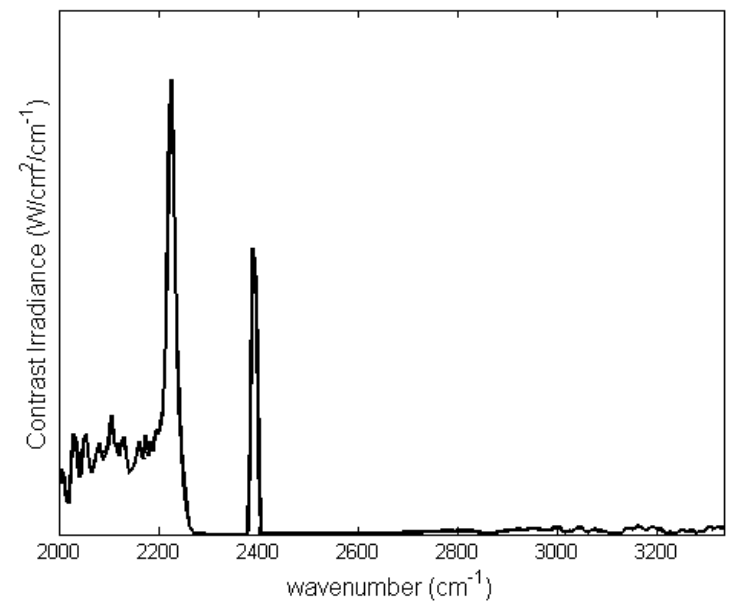

(a)

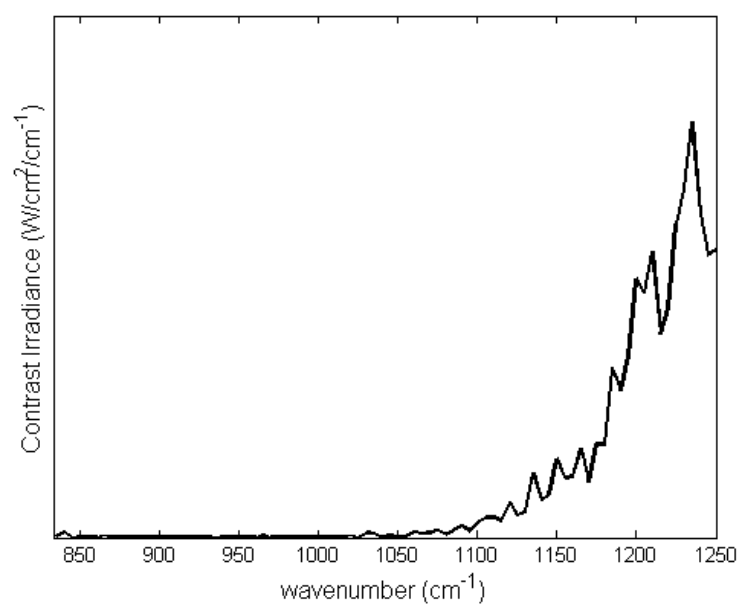

(b)

Figure 4 Contrast irradiance spectrum of an exhaust plume measured in (a) $3.0-5.0 \mu \mathrm{m}\left(3333-2000 \mathrm{~cm}^{-1}\right)$ and (b) $8.0-12.0 \mu \mathrm{m}\left(1250-833 \mathrm{~cm}^{-1}\right)$. 
Typical infrared sensors operate in the midwave $\left(3.0-5.0 \mu \mathrm{m}\left(3333-2000 \mathrm{~cm}^{-1}\right)\right)$ and longwave $(8.0-12.0 \mu \mathrm{m}(1250-$ $\left.833 \mathrm{~cm}^{-1}\right)$ ) atmospheric transmission windows. The wavelength band of $5.5-7.5 \mu \mathrm{m}\left(1333-1818 \mathrm{~cm}^{-1}\right)$, where the atmosphere absorbs all radiation, is outside this domain. However, the area around $4.1-4.5 \mu \mathrm{m}\left(2222-2439 \mathrm{~cm}^{-1}\right)$ is right in the middle of the $3.0-5.0 \mu \mathrm{m}$ domain. For the $8.0-12.0 \mu \mathrm{m}$ band the transmittance of the atmosphere is high, but the production of radiation by the plume is almost non-existent. Modern day cooled IR cameras use a smaller wavelength band of $8.0-9.0 \mu \mathrm{m}\left(1250-1111 \mathrm{~cm}^{-1}\right)$ or $8.0-10.0 \mu \mathrm{m}\left(1250-1000 \mathrm{~cm}^{-1}\right)$ and are able to detect some radiation produced by the plume. Figure 4 shows the contrast irradiance spectra of a plume, as seen by a sensor in the 3.0 $-5.0 \mu \mathrm{m}$ (a) and $8.0-12.0 \mu \mathrm{m}$ (b). These spectra are calculated for a combined speed $\mathrm{v}=15 \mathrm{~m} / \mathrm{s}, \theta=0^{\circ}$, an engine power of $100 \%$ and an atmosphere calculated with MODTRAN.

\subsection{Changing direction $\theta$}

The direction of the combined speed has an influence on the shape and size of the exhaust plume. One should remember that the combined speed vector is an addition of the ship's speed vector and the wind speed vector. The ship's heading is assumed to be $\theta=0^{\circ}$. The specific geometry of a ship is the main reason for different shapes and sizes exhaust plumes with changing wind direction.

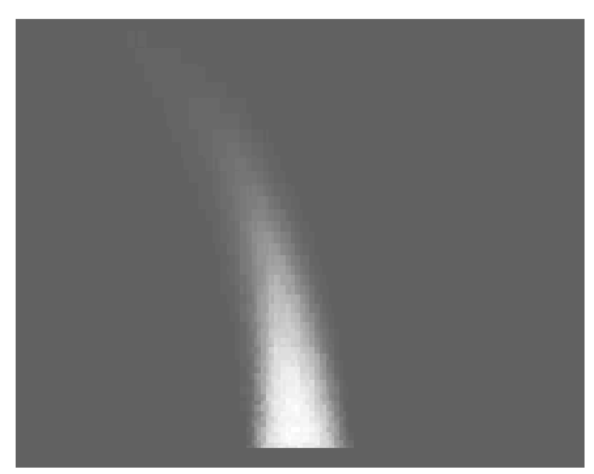

(a)

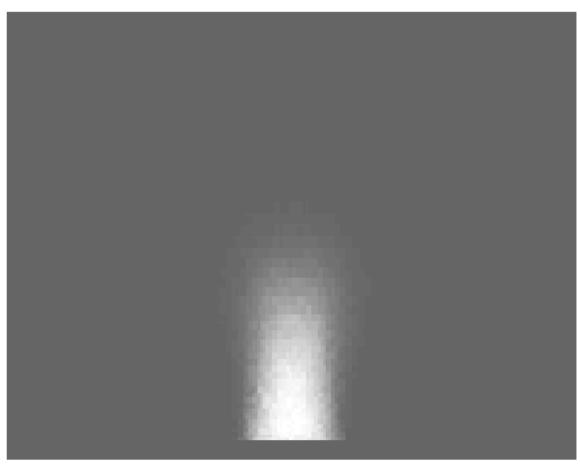

(c)

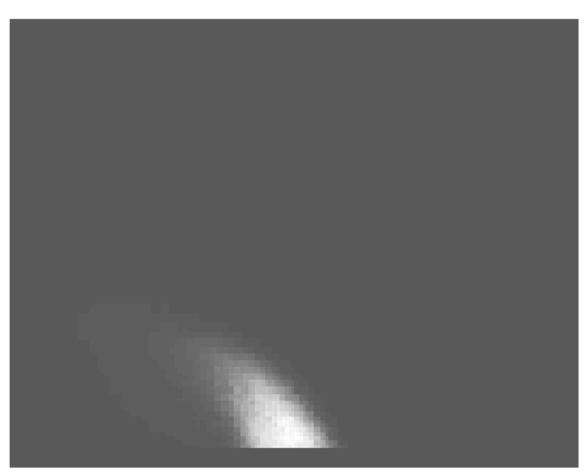

(b)

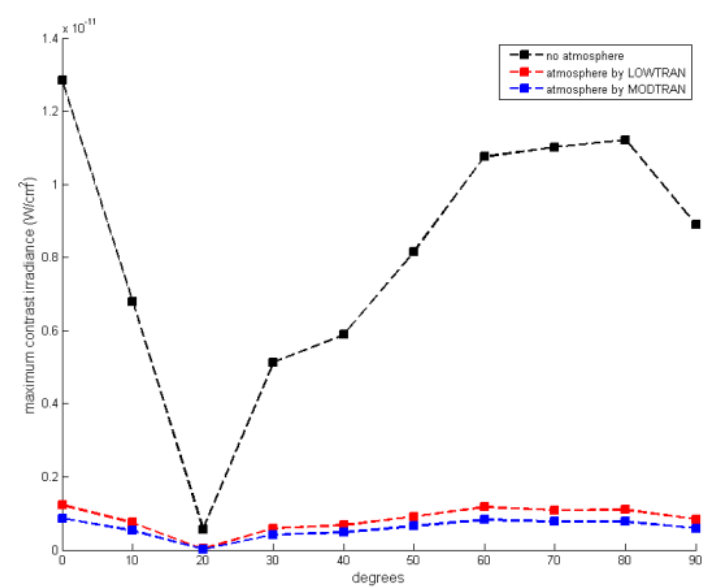

(d)

Figure 5 Radiance maps of an exhaust plume for (a) $\theta=0^{\circ}$, (b) $30^{\circ}$, and (c) $90^{\circ}, v=15 \mathrm{~m} / \mathrm{s}$, and engine setting of $100 \%$ calculated using MODTRAN in $3.0-5.0 \mu \mathrm{m}\left(3333-2000 \mathrm{~cm}^{-1}\right)$. (d) Maximum contrast irradiance vs. wind direction for no atmosphere (black line), calculation with LOWTRAN (red line) and MODTRAN (blue line) in 3.0 $-5.0 \mu \mathrm{m}\left(3333-2000 \mathrm{~cm}^{-1}\right)$. 
Figure 5 (a), (b), and (c) show the radiance maps of an exhaust plume, as registered by the sensor located $2 \mathrm{~km}$ behind the ship calculated, for a combined speed $\mathrm{v}=15 \mathrm{~m} / \mathrm{s}$, an engine power of $100 \%$, a wavelength band of $3.0-5.0 \mu \mathrm{m}$, and a direction $\theta=0^{\circ}, 30^{\circ}$, and $90^{\circ}$. The images shown here are calculated using MODTRAN to include the effects of the atmosphere between the plume and sensor. The shape of the plume does not change when the atmosphere is taken into account. Only the radiance that reaches the sensor is much lower when an atmosphere is taken into account. The shape of the plume is clearly different for the different directions (different angles of attack of the wind). The plume for $\theta=30^{\circ}$ stays much lower compared to the plumes for $0^{\circ}$ and $90^{\circ}$. Figure 5 (d) shows the maximum contrast irradiance as a function of the wind direction for the cases of no atmosphere (black line), an atmosphere calculated with LOWTRAN (red line) and MODTRAN (blue line). The minimum radiant intensity occurs in all cases at $\theta=20^{\circ}$ and the maximum occurs at $\theta=0^{\circ}$. The explanations for this behavior of the plume can be related to the geometry of the M-frigate and the streamlines on the ship's surface (not shown here).

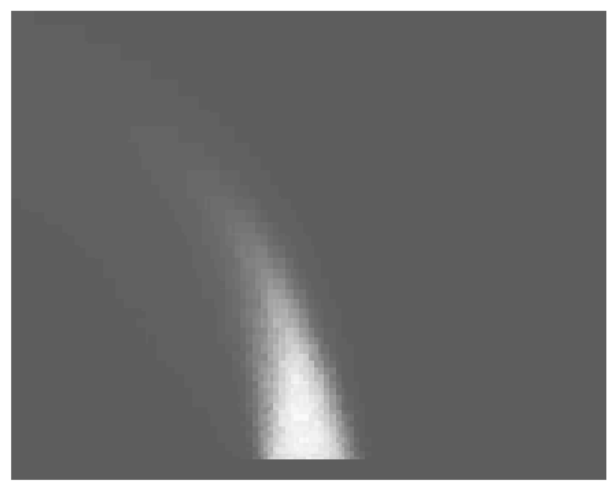

(a)

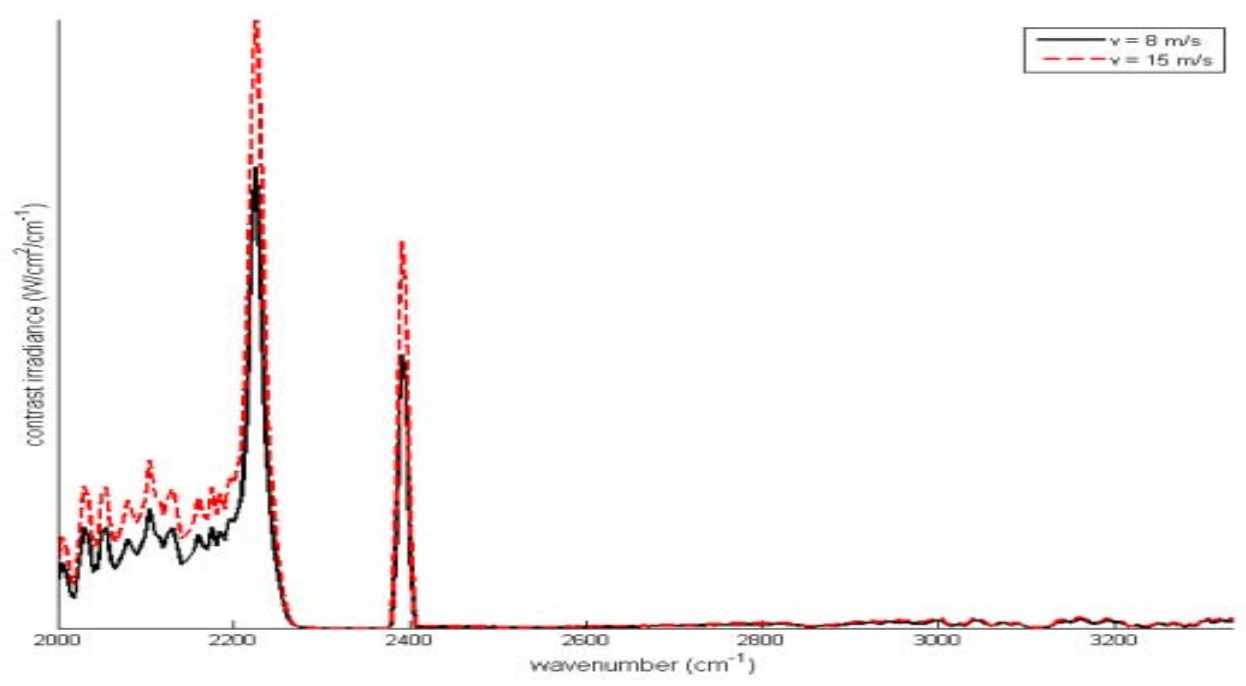

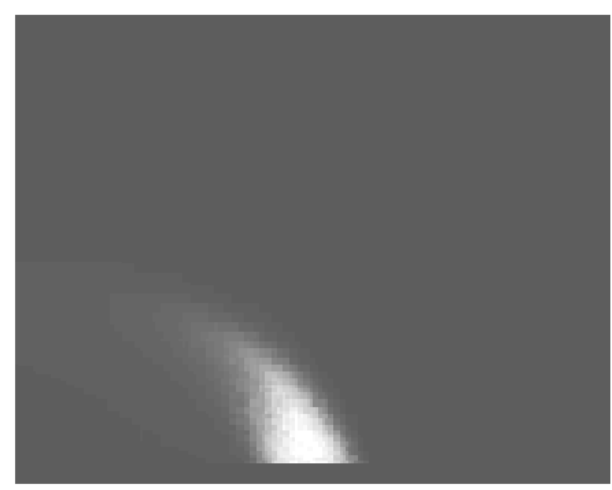

(b)

(c)

Figure 6 Radiance maps of exhaust plumes in the wavelength band of $3.0-5.0 \mu \mathrm{m}\left(3333-2000 \mathrm{~cm}^{-1}\right)$ for (a) $\mathrm{v}=8$ $\mathrm{m} / \mathrm{s}$ and (b) $\mathbf{v}=15 \mathrm{~m} / \mathrm{s}$. (c) Contrast irradiance spectra of the exhaust plumes calculated for $\mathrm{v}=15 \mathrm{~m} / \mathrm{s}$ (black line) and $v=8 \mathrm{~m} / \mathrm{s}$ (red line). Other parameters are $\theta=10^{\circ}$, an engine power of $100 \%$, and MODTRAN is used to include the atmosphere. 


\subsection{Different speed $\mathbf{v}$}

Another parameter which has an influence on the size and shape of the plume is the (combined) speed v. Figure 6 (a) and (b) shows the radiance maps of an exhaust plume calculated for two different (combined) speeds; (a) $\mathrm{v}=8 \mathrm{~m} / \mathrm{s}$ and (b) $\mathrm{v}$ $=15 \mathrm{~m} / \mathrm{s}$. These plumes are calculated for a direction $\theta=10^{\circ}$, an engine power of $100 \%$, a wavelength band of $3.0-5.0$ $\mu \mathrm{m}\left(3333-2000 \mathrm{~cm}^{-1}\right)$, and an atmosphere is included using MODTRAN. It is clear from this image that the plume stays lower, when the speed is higher, as could have been expected. In Figure 6 (c) the contrast irradiance spectra of the exhaust plumes for both speeds are plotted. A higher speed gives a smaller overall radiant intensity, which indicates a more efficient cooling of the plume by the surrounding air.

\subsection{Laser in the background}

An example of the use of the method introduced above, is indicated below. The calculations in this example give an estimate of the order and are not meant as exact numbers. Instead of a homogeneous background, a laser shines through the ship exhaust plume in the direction of the camera. The camera is located $2 \mathrm{~km}$ away from the plume and the laser is located $2 \mathrm{~km}$ away as well, but on the other side of the plume (see Figure 7 for a schematic drawing). The camera is located on the ground (height $=0 \mathrm{~m}$ ), the plume is at a height of $17 \mathrm{~m}$ and the laser is at a height of $34 \mathrm{~m}$.

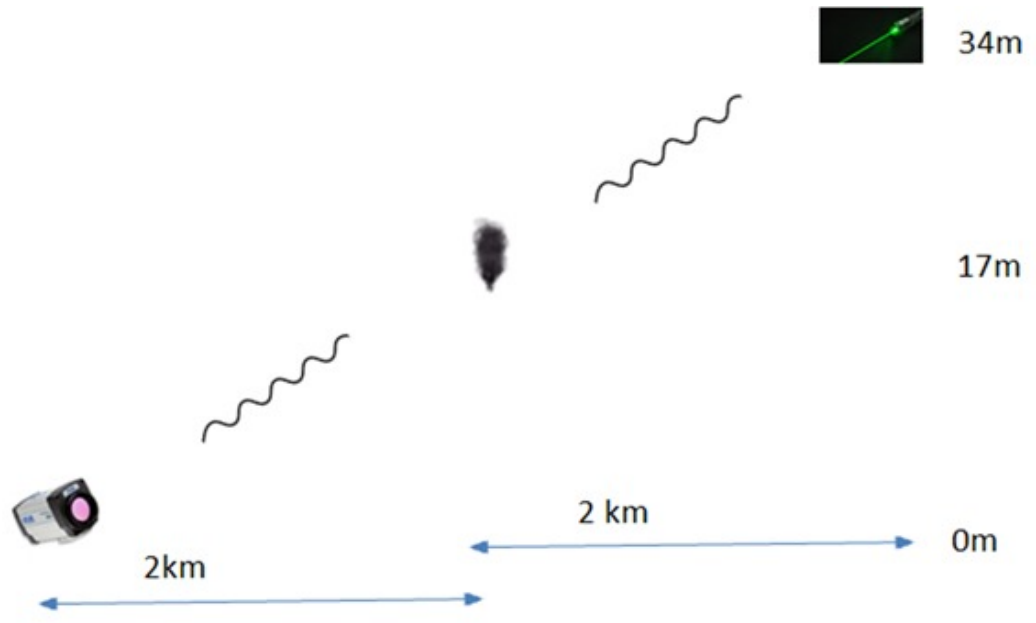

Figure 7 Schematic drawing of the set up used in the calculations.

The transmission of the exhaust plume is calculated using the modified version of NIRATAM. To calculate the total transmission from the laser to the sensor, the contribution of the two atmospheric paths outside the plume is calculated with MODTRAN. Three wavelengths are used for the laser; the first wavelength is in the blue spike at $\lambda=4.17 \mu \mathrm{m}(2395$ $\left.\mathrm{cm}^{-1}\right)$, the second wavelength is just outside this spike at $\lambda=4.29 \mu \mathrm{m}\left(2330 \mathrm{~cm}^{-1}\right)$, and the third wavelength is located in the LWIR domain at $\lambda=10.6 \mu \mathrm{m}\left(943 \mathrm{~cm}^{-1}\right)$. The transmission through the plume, $\tau_{\text {plume, }}$ is calculated at a speed $\mathrm{v}=15$ $\mathrm{m} / \mathrm{s}$, direction $\theta=0^{\circ}, 100 \%$ engine power, and no atmosphere present. The transmission through the atmosphere outside the plume is calculated for the slant paths laser - plume $(34 \rightarrow 17 \mathrm{~m}$ height $)$ and plume - camera $(17 \rightarrow 0 \mathrm{~m}$ height $)$. All these transmissions are given for the three wavelengths in Table 1.

Table 1 Transmission through plume, through the atmosphere on the path laser-plume, and on the path plume - camera for three different wavelengths and the total transmission.

\begin{tabular}{|l|l|l|l|l|}
\hline $\begin{array}{l}\text { Wavelength }[\boldsymbol{\mu m}] \\
\left.\text { (wavenumber }\left[\mathbf{c m}^{-1}\right]\right)\end{array}$ & $\begin{array}{l}\text { Transmission } \\
\text { through plume, } \\
\boldsymbol{\tau}_{\text {plume }}\end{array}$ & $\begin{array}{l}\text { Transmission } \\
\text { through atmosphere } \\
\text { from laser (34m) to } \\
\text { plume (17m), } \boldsymbol{\tau}_{\text {atm,34-17 }}\end{array}$ & $\begin{array}{l}\text { Transmission } \\
\text { through atmosphere } \\
\text { from plume (17m) to } \\
\text { camera (0m), } \boldsymbol{\tau}_{\text {atm,17-0 }}\end{array}$ & $\begin{array}{l}\text { Total } \\
\text { transmission }\end{array}$ \\
\hline $4.17 \mu \mathrm{m}\left(2395 \mathrm{~cm}^{-1}\right)$ & 0.9912 & 0.4243 & 0.4230 & 0.1779 \\
\hline $4.29 \mu \mathrm{m}\left(2330 \mathrm{~cm}^{-1}\right)$ & 0.6401 & 0.0 & 0.0 & 0.0 \\
\hline $10.6 \mu \mathrm{m}\left(943 \mathrm{~cm}^{-1}\right)$ & 1.000 & 0.5708 & 0.5674 & 0.3239 \\
\hline
\end{tabular}


Assuming the laser has an intensity $I$, it is possible to calculate the total intensity that reaches the camera, $I^{\prime}$, by:

$$
I^{\prime}=\tau_{a t m, 17-0} * \tau_{\text {plume }} * \tau_{a t m, 34-17} * I .
$$

For a laser at a wavelength of $4.17 \mu \mathrm{m}$ and intensity $I$, the camera registers an intensity of $0.1779 \mathrm{I}$. For a wavelength of $4.29 \mu \mathrm{m}$ the camera registers no intensity, since none of the radiation at this wavelength comes through the atmosphere. And for a wavelength of $10.6 \mu \mathrm{m}$ the camera registers an intensity of $0.3239 \mathrm{I}$. These calculations are performed using MODTRAN which uses a resolution of $5 \mathrm{~cm}^{-1}$, while the laser has a resolution of $1 \mathrm{~cm}^{-1}$. It would be better to use a code like HITRAN, which has this resolution. These calculations show that it is important to choose the correct wavelength for the laser.

To get a thorough understanding of the amount of light reaching the sensor, the power of the laser, the turbulence in the exhaust plume, and the presence of particulates (like sooth) in the plume and atmosphere should be taken into account.

\section{CONCLUSIONS}

A methodology is presented to calculate the IR signature of ship exhaust plumes. First a CFD model is used to calculate the 3D flow field of an exhaust plume, taking into account the structural geometry of the ship and the stack. Next gas radiation calculations are performed to determine the radiant intensity of the plume and the amount of background radiation through the plume. The output of these calculations can be used in a sensor performance model like EOSTAR, to assess the detection probability of the plume or objects behind it.

The radiant intensity and contrast irradiance were calculated for an exhaust plume under different circumstances. It was shown that the geometry of the platform has an influence on the size and shape of the exhaust plume. It was shown that a higher (combined) speed gives a spatially smaller and lower-rising exhaust plume. The temperature of the background has some influence on the contrast irradiance, especially in the $4.4-5.0 \mu \mathrm{m}$ band.

Finally we have shown that the radiation of a laser positioned at $2 \mathrm{~km}$ behind the plume can be registered on a camera 2 $\mathrm{km}$ in front of the plume. The wavelength of the laser needs to be chosen in such a way that the transmission through the exhaust plume and through the atmosphere is high enough. The power of the laser, turbulence in the exhaust plume, and particulates in the plume are not taken into account here.

\section{ACKNOWLEDGEMENTS}

The work in this paper was performed as part of the TNO research programme V1303 - Above Water Signatures sponsored by the Ministry of Defence of the Netherlands.

\section{REFERENCES}

[1] Sjöqvist, L., "Laser beam propagation in jet engine plume environments - a review", Proc. SPIE 7115 (2008).

[2] Andrews, L.C. and Phillips, R.L., "Laser Beam Propagation Through Random Media", SPIE Press (2005).

[3] Ric H. Schleijpen, "Laser pointing in the vicinity of jet engine plumes", SPIE Vol. 7483, paper13, Technologies for Optical Countermeasures VI (2009).

[4] Noah, M., Kristl, J., Schroeder, J., "NIRATAM - NATO Infrared Air Target Model”, Proc. SPIE 1479, 275 282 (1991).

[5] Kunz, G.J., Moerman, M.M., van Eijk, A.M.J., Doss-Hammel, S.M., and Tsintikidis, D., "EOSTAR : An electrooptical sensor performance model for predicting atmospheric refraction, turbulence and transmission in the marine surface layer", Proc. SPIE 5237, 81-92 (2003).

[6] Van Eijk, A.M.J., Degache, M.A.C., Tsintikidis, D., and Hammel, S., "EOSTAR Pro: a flexible extensive library to assess EO sensor performance", Proc. SPIE 7828 (2010).

[7] Van Eijk, A.M.J., Degache, M.A.C, and de Lange, D.J.J., "The EOSTAR model suite" in International Symposium on Optronics in Defense and Security, OPTRO 2010, 3-5 February, Paris, France. (2010).

[8] Neele, F.P., "Infrared ship signature prediction, model validation and sky radiance", Proc. SPIE 5811, 180-187 (2005). 
[9] Schwering, P.B.W., "IRST evaluation methodologies: Maritime infrared background simulator”, Proc. SPIE 6206 (2006).

[10] Kunz, G.J., "A bulk model to predict optical turbulence in the marine surface layer", TNO-FEL Report Nr. FEL96-A053, TNO Physics and Electronics Laboratory, The Hague, The Netherlands (1996).

[11] Penelon, T., Calmet, I., and Mironov, D.V., "Mircometeorological simulations over complex terrain with SUBMESO: a model study using a novel preprocessor". Int. J. Env. Poll. 16, 583-602 (2001).

[12] Vaitekunas, D.A., Sideroff, Ch., and Moussa, Ch., "Improved signature prediction through coupling of ShipIR and CFD”, Proc. SPIE 8014 (2011).

[13] ANSYS Inc., "Fluent 13 Documentation", 2010.

[14]Ludwig, C.B., Malkmus, W., Raerdon, Y.E., and Thomson, J.A.L., "Handbook of Infrared Radiation from Combustion Gases", National Aeronautics and Space Administration, NASA-SP 3080 (1973).

[15] Fair, M.L., "NIRATAM Software User Guide", TR/DERA/WSS/WX3/TR980154/1.0 (1998).

[16] Kunz, G.J., Moerman, M.M., and van Eijk, A.M.J., "ARTEAM : Advanced Ray Tracing with Earth Atmospheric Models", Proc. SPIE 4718, 397-404 (2002). 